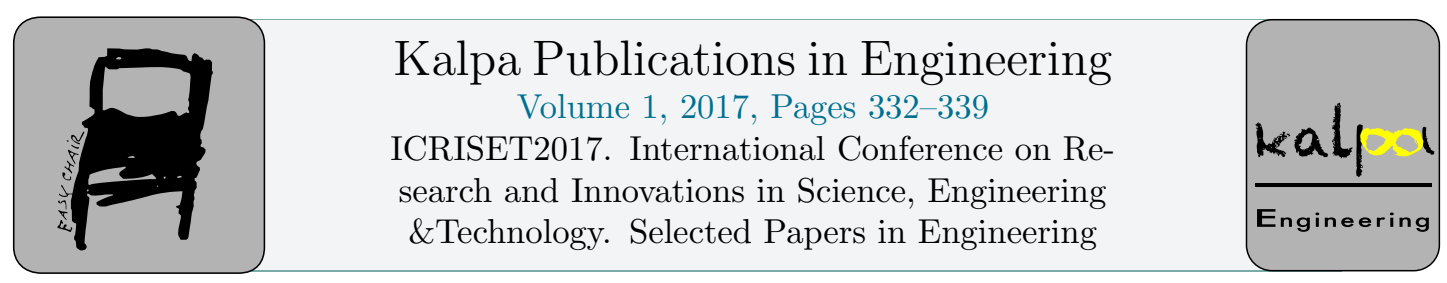

\title{
Develpoment of Experimental Quad Copter for Laboratory Demonstration
}

\author{
Sagar Vala ${ }^{1}$, Varun Dave ${ }^{2}$, Mrs. Dharita K. Patel ${ }^{3}$ and Dr. Rashesh P. \\ $\mathrm{Mehta}^{4}$ \\ ${ }^{1}$ Research Scholar, ${ }^{2}$ Research Scholar, ${ }^{3}$ Assistant Professor, ${ }^{4}$ Associated Professor \\ ${ }^{1,2,3,4}$ Electrical Engineering Department, BVM Engineering College, V V Nagar, Anand, India \\ ${ }^{1}$ sagarvala1996@gmail.com, 2varunt.8319@gmail.com, \\ ${ }^{3}$ dkpatel@bvmengineering.ac.in, ${ }^{4}$ rpmehta@bvmengineering.ac.in
}

\begin{abstract}
In this project a scheme has been initiated to develop self-directed land Flying robot using software and tools. A quad-rotor helicopter (i.e. QUADCOPTER) is an aircraft whose lift is generated by four propellers driven by rotors. The control mechanism of such an aircraft consists of four Brushless DC motors whose speeds are varied relative to each other. In order to allow for stable flight of the quad-rotor, the control system employed must be fastidious. Uncontrolled flight of a quad-rotor would be practically impossible by one operator, as the system demand constant adjustment of four motors simultaneously. Generally the speed and rotation of a quadcopter is adjusted with the help of remote control. However in order to get rid of bulky remote controls speed and rotation can be controlled by the use of a sensor which detects the motion of hand and will change its direction accordingly
\end{abstract}

Key words: quadcopter; motion control; accelerometer; manoeuvrable

\section{Introduction}

Human beings are captivated by levitation. One possible reason could be our threedimensional world. However their movement is mainly confined in two dimensions. It is quite difficult for human beings to surmount their biological boundaries. This leads to the invention of machines that enable them to move in three-dimensional space, e.g., airplanes and helicopters.

The main aim of this project is to design and fabricate a small, robust and highly manoeuvrable autonomous flying robot which can withstand any weather conditions. At present due to hardware and software limitations, the control of similar technologies is done at low frequencies [1]. 
However it leads to improbability in position control and instability during fast tactics. The new proposed flying platform offers a $2.4 \mathrm{GHz}$ control frequency and motor update rate, in arrangement with powerful brush-less DC motors in a light-weight package. Moreover the control of the direction and speed is done by motion control which enables the user to control without any extra equipment, and due to higher frequency the cost of this equipment will be economical.

\section{Research Analysis}

\section{The fundamental components of quadcopter are:-}

\subsection{Motor and propeller}

\subsection{Microcontroller}

\subsection{Electronic speed controller (ESC)}

\subsection{Gyroscope \& Accelerometer}

\subsection{Transmitter \& Receiver}

\subsection{Motors}

The most important component of this project is motor without which the quadcopter cannot work. There is availability of many motors in market. Hence choice of proper motor is necessary for the successful working of quadcopter. Brushless DC motors and servo motors are the two most common types of motors used in quadcopter. Since the main purpose of a quadcopter is having flexibility in its direction control and efficiency so with the view of that both these motors were compared. The conclusion derived from the above comparison was in favour of BLDC motor. Thus BLDC motor as shows in Fig. 1 was chosen instead of servo motor because of its simplicity in direction control, high dynamic response and noiseless durable operation.

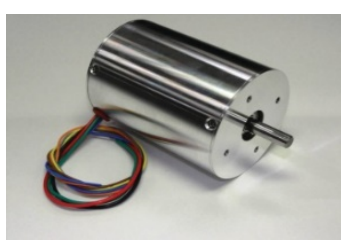

Fig1. BLDC motor 


\subsection{Microcontroller}

ATmega328P has been chosen for this project because of its features like 14 digital input/output pin, $16 \mathrm{MHz}$ quartz crystal frequency and mainly providing $6 \mathrm{PWM}$ outputs which enable to use for the input pulse of motor through electronic speed controller(ESC).

\subsection{Electronic Speed Controller (ESC)}

Electronic speed controller is a power electronic device used in a quadcopter as an interface of motor and microcontroller. It is an electronic circuitry which can vary the motor's speed, direction and can also function as a dynamic brake. ESC of 30A has been used in this project.

\subsection{Accelerometer}

Accelerometer is the equipment which measures acceleration and provide feedback coordinates to the controller accordingly. It is a type of motion sensor. Eventually with the use of this achieved coordinates we are able to change the direction and speed of the motor in quadcopter. Despite the option of gyroscope we choose accelerometer because gyroscope limits its application to maintain the stability of an object only.

\subsection{Transmitter \& Receiver}

A radio transmitter is an electronic device which can convert electric power from a battery or any other electric source to a radio frequency alternating current. A radio receiver is electronic circuit that receives its input from an antenna, uses electronic filters to discrete a wanted radio signal from all other signals picked up by this antenna, amplifies it to a level suitable for further processing, and finally converts over demodulation and decoding the signal into a form usable for the consumer, such as sound, pictures, digital data. This project has used a $2.2 \mathrm{GHz}$ radio frequency with six different channels. Fig. 2 shows the block diagram of a quadcopter.

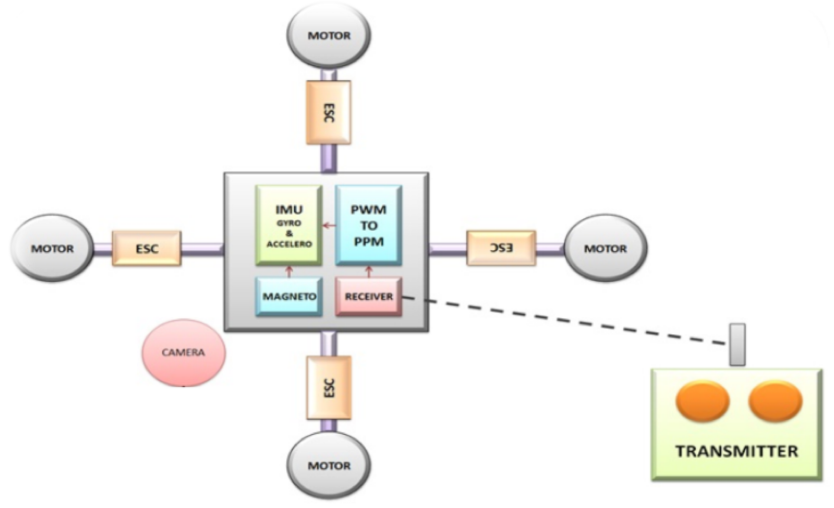

Fig. 2. Block diagram of a quadcopter

Flow of signal received can be written as: receiver $->$ Microcontroller $->$ Gyroscope $->$ Electronic speed controller $->$ Motor 


\section{Flight Mechanism}

\subsection{Airplane flight control}

Before understanding the flying scheme of quadcopter let us see the flying mechanism of airplane since quadcopter uses the same technique in flying.

In airplane there are three main axis by which we can levitate or control it. By changing the values of different axis, controlling of the plane in the suitable direction can be achieved [3]. This axis's are called as pitch, roll and yaw which have been shown in fig. 3 . Thus by changing the magnitude of respective axis we can control the flight. Minimum two or more axis is simultaneously used to control the flight. For example if we want the airplane to turn left than the change in the magnitudes of yaw and roll axis are done. A similar technique is used to change the direction of quadcopter [2].

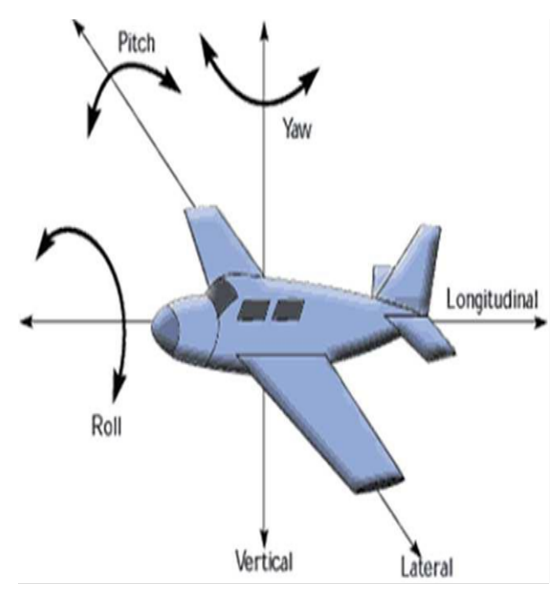

Fig. 3. Axis of airplane 


\subsection{Thrust mechanism}

The fundamental requirement of a quadcopter to fly is thrust. For giving equivalent and balanced thrust we need to use two pairs of motors rotating in opposite directions. The first pair of motor for clockwise (C.W) direction and other for counter clockwise direction (C.C.W). The main reason behind this counter rotation is to keep the centre of gravity of quadcopter at the centre of the frame. Let us understand this with the help of an example. Suppose we have a floating bar which is supported at its centre. Now if we want to balance this bar more perfectly than we need to balance the forces exerting on both the ends of the bar. There are two methods to do this. The first or the basic method is to apply two forces in the same direction at both ends. However the bar can also be balanced if we apply two forces of same magnitude but in opposite directions at both the ends of the bar. The later method is what has been applied in quadcopter as shown in fig. $4[2]$.

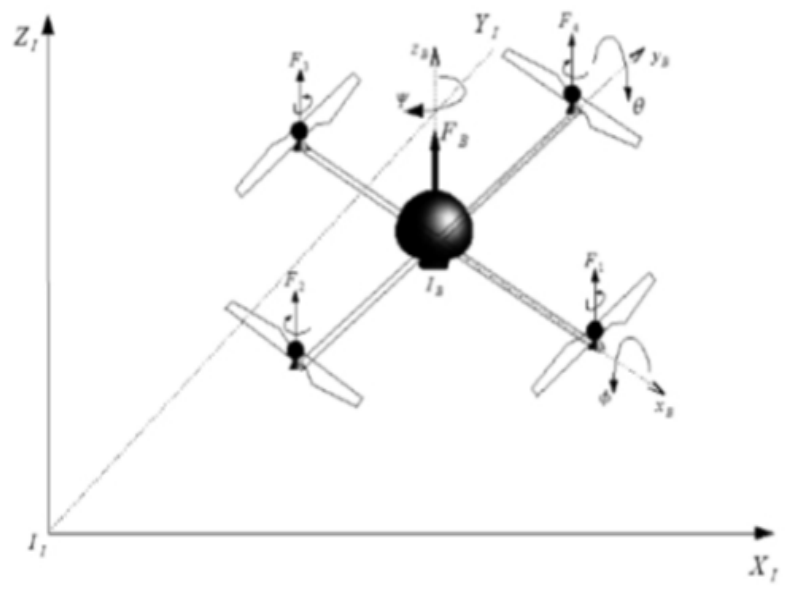

Fig. 4. Direction of motors

\subsection{Direction Control}

In the present quadcopter we don't have flips are same as in airplane so to change the values of pitch, roll and yaw axis for changing the direction, we need to change the speed of respective motor. The quadcopter adjust its altitude by having equal thrust from all the four motors or say it will be in stable position. Now in order to adjust its yaw axis quadcopter has to apply more thrust to the diagonal motors which are rotating in same direction. And quadcopter can control its pitch and roll by applying more thrust to one motor and less to another diagonal motor. Fig. 5 shows the method of direction control in a quadcopter.
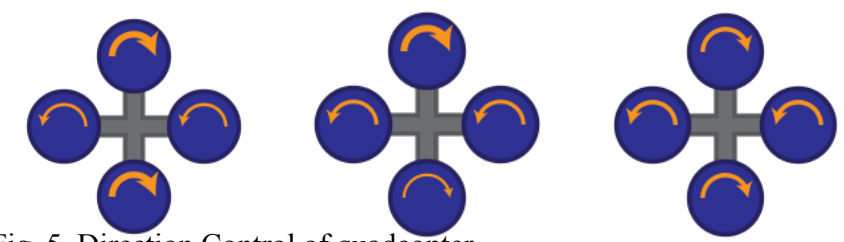

Fig. 5. Direction Control of quadcopter 


\section{Motion Control}

\subsection{Accelerometer}

Accelerometer is simply a device which can detect any motion or acceleration and produce output across its terminals in the form of electrical signal. The output of accelerometer can be analog or digital. There are different types of accelerometer like piezoelectric accelerometer, Thermal accelerometer, and capacitive accelerometer. The piezoelectric accelerometer will produce output according to the change in pressure or stress applied on the body. Not only this but another piezoelectric accelerometer work on the principle of change in the resistance of electric material which is under stress. Now to get the exact position we need to integrate the data of accelerometer twice, since first integration gives us velocity and second integration will give acceleration. Thus we are able to get the change in direction, acceleration and the position movement. There are dual axis and tri axis accelerometer. We have used tri axis accelerometer has been used in this project since it gives better precision and accuracy than the dual axis accelerometer. Fig. 7 shows the model of a quadcopter.
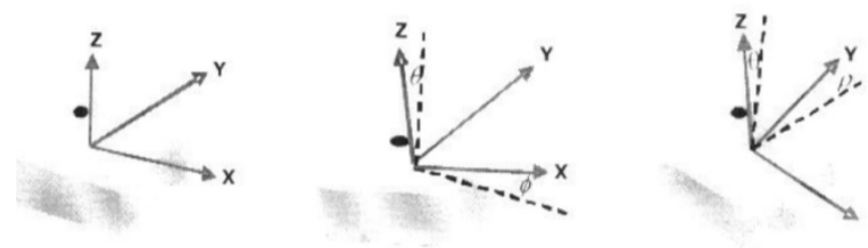

Fig. 6. Axis of accelerometer

Fig. 6 shows the change in angle accordingly for a tri axis accelerometer. Here $\mathrm{X}$ and $\mathrm{Y}$ axis can sense the change in potion whereas $\mathrm{Z}$ axis can be used for both sensing the change in position and to improve the precision. Here $Q$ and $\Theta$ are the angles of accelerometer with respect to the reference axis [4]. 


\begin{tabular}{l|l|l|l|l|l}
\hline $\begin{array}{l}\text { Movement } \\
\text { of hand }\end{array}$ & \multicolumn{3}{ll}{ Input to the controller } & Direction \\
\hline & D3 & D2 & D1 & D0 & \\
\hline & 0 & 0 & 0 & 0 & $\begin{array}{l}\text { Stop } \\
2.0 \mathrm{GHz}\end{array}$ \\
\hline & 0 & & & & \\
\hline
\end{tabular}

1

Table 1. Motion Control Analysis

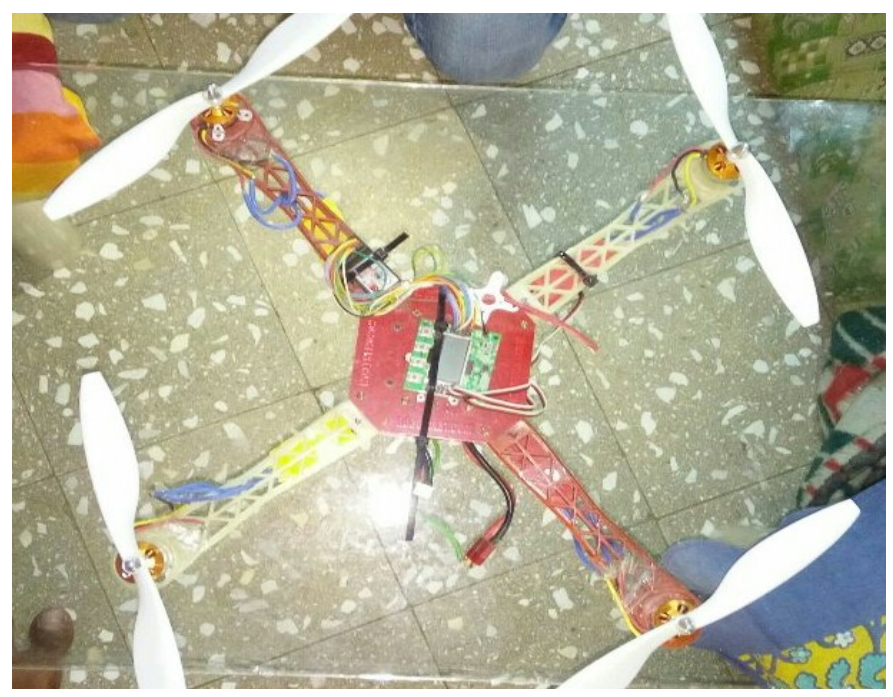

Fig. 7. Model of Quadcopter 


\subsection{Hand controlled coordination}

As we have seen that with the accelerometer we can turn any physical movement into its equivalent electrical signal, we are here converting the movement of our hand into its equivalent electrical signal. This signal is transmitted through a $2.2 \mathrm{GHz}$ frequency channel to the receiver of quadcopter. The change in the direction and frequency according to the movement of hand is shown in the Table 1.

\section{Conclusion}

As per this design, the quadcopter can function based on the movement of hand. It employs the principle of motion control with the help of accelerometer. It attains an appropriate lift based on a typical flying mechanism and can be used for investigation purposes. Moreover it provides flexibility in its movement. It can also be used to provide surveillance at night with the help of infrared cameras. This system can further be enhanced for future prospects.

\section{References}

[1] A. Olivares-Mendez and Luis Mejias and Pascual Campoy and Ignacio Mellado-Bataller "Quadcopter See and Avoid Using a Fuzzy Controller M. " " in Australian Research Centre for Aerospace Automation (ARCAA) Queensland University of Technology Brisbane, Australia.

[2] Mohd Khan "Quadcopter Flight Dynamics" International Journal Of scientific and Technology Research volume 3, issue 8, August 2014 ISSN 2277-8616.

[3] Mark W. Mueller and Raffaello D'Andrea "Stability and control of a quadrocopter despite the complete loss of one, two, or three propellers" 2014 IEEE International Conference on Robotics \& Automation (ICRA)Hong Kong Convention and Exhibition Centre May 31 June 7, 2014. Hong Kong, China.

[4] Pankaj Singh Bhavik Gupta, Ankit Patel, Anurag Kumar, Mohit Ujjwal "Autonomous Intelligence Surveillance Quadcopter" International Journal of Engineering Research \& Management Technology March- 2015 Volume 2, Issue-2 ISSN: 2348-4039. 